\title{
Total oder totalitär?
}

\section{Karl Barths Verständnis von Vertrauen in der I. These der Barmer Theologischen Erklärung (1934)}

Marcel Egli

Ich bin der Weg und die Wahrheit und das Leben; niemand kommt zum Vater denn durch mich. (Joh 14,6)

Wahrlich, wahrlich, ich sage euch: Wer nicht zur Tür hineingeht in den Schafstall, sondern steigt anderswo hinein, der ist ein Dieb und Räuber.

Ich bin die Tür;

wenn jemand durch mich hineingeht, wird er selig werden. (Joh 10,1.9)

Jesus Christus, wie er uns in der Heiligen Schrift bezeugt wird, ist das eine Wort Gottes, das wir zu hören,

dem wir im Leben und im Sterben zu vertrauen und zu gehorchen haben.

Wir verwerfen die falsche Lehre, als könne und müsse die Kirche als Quelle ihrer Verkündigung außer und neben diesem einen Worte Gottes auch noch andere Ereignisse und Mächte, Gestalten und Wahrheiten

als Gottes Offenbarung anerkennen.

(I. These der Barmer Theologischen Erklärung)

\section{I.}

Zur Zeit der Abfassung dieser hier abgedruckten These war die Aufgabe von Theologie und Kirche strittig geworden. Theologie und Kirche haben formal betrachtet ihre Aufgabe darin, sich um das Verstehen des Wortes Gottes zu bemühen. In inhaltlicher Hinsicht haben sie dieses in eine adäquate, verständliche Rede vom Menschen zu überführen. Dies ist eine nicht notwendigerweise lösbare Aufgabe, da es sich für den Menschen nicht von selbst versteht, was von ihm zu sagen und wie von ihm zu reden ist. Denn anders als ein Gegenstand, der klar umrissen vor einem erscheint, zeigt sich der Mensch sich 
und anderen nie in klaren Konturen ${ }^{1}$. In ihrem Bemühen um die Rede vom Menschen haben Theologie und Kirche dieses Wesen des Menschen zu respektieren und können deshalb weder Vorschriften formulieren, wie der Mensch sein soll, noch Wunschvorstellungen entwerfen, wie er sein könnte, sondern bloß versuchen, ihn als den anzureden, als der er sich abgesehen von eigensinnigen Vorannahmen immer wieder in Hinsicht auf das Wort Gottes erst erweist. In welcher Gestalt theologisch und kirchlich vom Menschen die Rede ist, bleibt dabei abhängig vom Wahrheitsbewusstsein und den Sprachgewohnheiten, die jeweils üblich sind. Zwar ist, wer der Mensch konkret ist, nicht von diesen Umständen abhängig, dennoch kann nicht immer in derselben, sondern muss in einer unter jeweiligen Umständen nachvollziehbaren Weise vom Menschen geredet werden, damit vom Menschen verständlich die Rede ist.

Die Rede vom Menschen hat also einen eigentümlich unbestimmbaren, sich der Bestimmbarkeit entziehenden Gehalt, während die Artikulationsweisen, mittels derer vom Menschen die Rede ist, ihrerseits immer bestimmbar sind. Die Bedingungen adäquater Rede vom Menschen lassen sich (nicht ausschließlich, aber auch) dadurch ermitteln, dass konkrete Redeweisen untersucht werden, durch die der Mensch anzusprechen versucht wird. Bemerkenswert sind dabei gerade jene Formen, die sich nicht bewähren, sondern von den angeredeten Menschen als inadäquat, un- oder missverständlich zurückgewiesen werden, weil sie für sie nicht ansprechend sind. Ein historisches Datum, an dem im kirchlichen Bereich solcher Widerspruch artikuliert wurde, steht am Anfang der nationalsozialistischen Herrschaft in Deutschland zu Beginn der 1930er Jahre. Die Bewegung der «Bekennenden Kirche〉 reagierte damals auf den Anschluss der Deutschen Christen an die nationalsozialistische Ideologie. Sie hatte das Ansinnen der Deutschen Christen, sich dem nationalsozialistischen System unterzuordnen, als theologisch und kirchlich unsachgemäß zurückgewiesen und deshalb ihrerseits für die evangelischen Kirchen in Deutschland den status confessionis, den Zustand der Bekenntnisnot, ausgerufen. Im Rückblick schreibt dazu der führende Kopf jener Bewegung: «Die Kirche hatte auf einmal nur noch die Wahl, entweder sich selbst als Kirche aufzugeben oder

\footnotetext{
1 Im Hinblick auf die menschliche Selbsterkenntnis stellt J.-L.Marion,L'indéfinissable ou la face de l'homme, in: ders., Certitudes négatives, Paris 2010, 21-86, 25, deshalb fest: «Nous connaissons quelque chose que nous pouvons bien entendu nommer nous-même; je me connais donc, mais précisément je ne me connais qu'en tant que connu, jamais en tant que connaissant, donc jamais en tant que je suis spécifiquement celui qui pense ce qui, sans lui, resterai non pensé, ni connu.»
} 
aber Kirche zu bleiben, dann aber als solche zu kämpfen, zu bekennen, zu leiden ${ }^{2}$.

Die lutherischen, reformierten und unierten Kirchenvertreter und Theologen, die sich zur Bewegung der Bekennenden Kirche zusammengeschlossen hatten, versammelten sich deshalb am 31. Mai 1934 in Barmen/Wuppertal zu einer Bekenntnissynode, um ihre Position zu erörtern und in einem Bekenntnistext zum Ausdruck zu bringen. In einer ¿Erklärung» wurde den Deutschen Christen widersprochen. Es wurde zur Sprache gebracht, welche Ansichten $\mathrm{zu}$ verwerfen und was demgegenüber als verantwortbare Grundlage christlicher Lehre zu vertreten ist. Entstanden ist die Theologische Erklärung zur gegenwärtigen Lage der Deutschen Evangelischen Kirche>. Verfasst wurde sie weitgehend von dem damals in Bonn lehrenden reformierten Schweizer Theologen Karl Barth. Diese ¿Erklärung〉 nimmt wesentliche Positionen von Barths Theologie auf (insbesondere die Zentralstellung der Christologie), weshalb im Folgenden oft Barth selbst das Wort haben wird. Für die Frage nach dem theologischen Verständnis von Vertrauen ist die Barmer Erklärung deshalb interessant, weil sie Möglichkeitsbedingungen echten, tragfähigen Vertrauens nennt. Mein Vorschlag ist es, diese Bedingungen als Regeln adäquater Rede vom Menschen zu verstehen, wobei sich die I. Barmer These mit der Frage befasse, woran sich die adäquate Rede vom Menschen zu orientieren habe.

II.

Was stand in jenem Streit zwischen Bekennender Kirche und Deutschen Christen eigentlich auf dem Spiel? Im Zuge des ¿Überhandnehmens〉 der Deutschen Christen innerhalb der Strukturen der Deutschen Evangelischen Kirche, d.h. innerhalb von Kirchenleitungen und Pfarrerschaft, weitete sich die Herrschaft des Staates auf die Belange der Kirche ${ }^{3}$ aus. Diese Entwicklung provozierte in den Kreisen der Bekennenden Kirche den status confessionis. Strittig geworden war das Verhältnis der Kirche zum Staat. Die

2 K. Barth, Kurze Kommentierung des ersten Satzes der Theologischen Erklärung der Barmer Synode vom 31. Mai 1934, in: Texte zur Barmer Theologischen Erklärung. Mit einer Einleitung von E. Jüngel und einem Editionsbericht herausgegeben von M. Rohkrämer, Zürich ${ }^{2} 2004,67-87,79$.

3 Nach den Richtlinien der Deutschen Christen Ende 1933 «erkennt die Kirche im Totalitätsanspruch des nationalsozialistischen Staates den Ruf Gottes zu Familie, Volk und Staat» (A. Burgsmüller/R. Weth [Hg.], Die Barmer Theologische Erklärung. Einführung und Dokumentation, Neukirchen-Vluyn 1984, 38). 
Kirche war nicht mehr souveränes Gegenüber zum Staat, sondern wurde ihm gleichgeschaltet und seiner Ideologie unterworfen. Die offizielle Kirche hatte damit aus der Sicht der Bekennenden Kirche die in ihrer ausschließlichen Grundorientierung am Evangelium begründete Freiheit und damit nichts weniger als ihr eigentliches Wesen preisgegeben.

«Die Deutschen Christen und ihr Regiment bedrohen nicht irgend etwas in der Deutschen Evangelischen Kirche durch die Geltendmachung andrer, fremder Voraussetzungen, (sondern sie bedrohen) mit ihrer theologischen Voraussetzung sie selbst» ${ }^{4}$.

Dem Evangelium Jesu Christi wurde eine staatliche Doktrin und die Orientierung an Rasse und Herkunft vor- und übergeordnet. Damit galt in der Kirche nicht mehr nur bloß ein anderer Buchstabe, sondern wehte auch ein anderer, ihr fremder Geist. Die Kirche, die sich ihrem Wesen nach als Leib Christi versteht, wurde zur Dienerin zweier Herren, Christi sowie des deutschen States und dessen Doktrin. Für die Bekennende Kirche stellte sich die Frage:Wodurch und in welcher Weise kann die Alleinherrschaft Jesu Christi über die Kirche in aller Deutlichkeit wieder zur Geltung gebracht und damit die adäquate Rede vom Menschen wieder ermöglicht werden? Die Barmer Synode nahm sich dieser Frage an und bezog eine Gegenposition zu den Deutschen Christen, indem sie dazu aufforderte, sich neu auf das in der Bibel bezengte Wort Gottes zu besinnen. Ihrem eigenen Anspruch nach war das, was sie unternommen hat, nicht revolutionär, sondern bloß ein Bekennen des alten Glaubens unter neuen Umständen.

Nach Barth ist ein Bekenntnis «der Akt, in welchem die Kirche sich zwischen der Heiligen Schrift und einem Zustand, in welchem sie sich befindet, sich aufs neue bestimmt auf das, was Kirche ist» ${ }^{5}$. Ein Bekenntnis ist damit vergleichbar dem Akt der Verkündigung. Es ist wie auch jede gottesdienstliche Verkündigung den biblischen Berichten verpflichtetes Zeugnis von dem aller Gegenwart gegenwärtigen Wort Gottes (Jesus Christus). Es ist keine bloß erfundene, unbegründete, sondern an das gegenwärtig wirkende Wort Gottes selbst gebundene Antwort auf die Frage «was glauben wir als

\footnotetext{
4 K. Barth, Die theologische Erklärung der Barmer Bekenntnissynode. Fragment vom Sommer oder Frühherbst 1934, in: Texte zur Barmer Theologischen Erklärung, 25-58, 52 .

5 K. Barth, Kurze Erläuterung der Barmer Theologischen Erklärung.Vortrag vor der Evangelischen Bekenntnisgemeinschaft Bonn am 9. Juni 1934, in: Texte zur Barmer Theologischen Erklärung, 9-24, 12.
} 
Kirche?), (was haben wir als Kirche und als Glieder dieser Kirche zu tun?), und damit insgesamt auf die Frage (wer sind wir eigentlich?). Das Bekenntnis hat also die Funktion, dem gegenwärtig wirkenden Wort Gottes nachdenkend, zu klären, was als Norm, Verpflichtung und Gehalt christlicher Identität zu gelten hat. Diese Klärung geschieht stets unter Aufnahme, aber eben auch Kritik und wenn nötig Ablehnung geltender Wahrheitsansprüche. Die sechs Thesen der Barmer Theologischen Erklärung sind deshalb an ihre Zeit gebunden. Sie sind «in keiner Weise zu vergleichen mit dem apostolischen Glaubensbekenntnis oder auch nur mit der Confessio Augustana oder der Katechismus, sondern es geht in ihnen nur um Antworten auf Fragen, die uns jetzt, in diesem Jahr 1934 gestellt sind $»^{6}$.

III.

Die Norm der Verkündigung des Evangeliums war in dringlicher Weise grundsätzlich strittig geworden. Es ist bedeutsam, dass es dabei nicht bloß um einen akademischen Fachdiskurs ging, sondern es bestand eine kirchenpolitische Herausforderung. Das Verständnis der Kirche und das Selbstverständnis ihrer Glieder war in der kirchlichen Öffentlichkeit verantwortlich darzulegen und gegen eine innerhalb der Kirche wirksam gewordene Ideologie zu verteidigen. In der Meinung Barths hatte man sich dafür neu auf die Bibel zu besinnen; eine «natürliche Theologie» hätte nicht die nötige Kraft, um den Grundsätzen der Deutschen Christen theologisch fundiert zu widersprechen. Denn sie orientiert sich an sichtbaren Werten (am religiösen Gefühl des Einzelnen, an Herkunft, Rasse, Staatsordnung usf.) und steht dabei offensichtlich auf derselben Argumentationsebene wie die zu bekämpfende Position. Die Barmer Bekenntnissynode verständigte sich auf die Orientierung am den Menschen durchaus angehenden, aber dennoch unsichtbaren, unverfügbaren Wort Gottes (Jesus Christus), das die Texte der Bibel zur Sprache bringen. Die synodale Klärung mündete in die Position, dass Jesus Christus - und kein anderes Wort - das Wort Gottes ist, das zu verstehen und dem $\mathrm{zu}$ vertrauen und $\mathrm{zu}$ gehorchen ist. Anders als mit den Weltverhältnissen beliebig wechselnde Werte und Worte und dem Menschen gegenüber ignorante natürliche Ordnungen, verlangt dieses den Menschen ansprechende Wort Gottes, «im Leben und im Sterben die Totalität des Menschen, der sich ihm ergeben will» ${ }^{7}$.

6 K. Barth, Kurze Erläuterung, $17 \mathrm{f}$.

7 K. Barth, Kurze Erläuterung, 18. 
Und diese totale Inanspruchnahme kann den Menschen als Menschen bewahren, während er in der totalen Versachlichung der Perspektive natürlicher Theologie (oder einer Ideologie) verlorenzugehen droht. Der erste Satz der Barmer Theologischen Erklärung

«schließt die natürliche Theologie von der Verkündigung aus. Nicht in der Meinung und Absicht, sie in sich und als solche zu verwerfen, wohl aber in der Meinung und Absicht, daß sie neben und außer dem Worte Gottes, wenn es darauf ankommt, $z u$ sagen, wem wir im Leben und im Sterben zu vertrauen und zu gehorchen haben, keinen Sinn und Bestand haben kann. Als Gottes Offenbarung, als Norm und Inhalt der im Namen Gottes auszurichtenden Botschaft, können die Größen, auf die die natürliche Theologie sich zu beziehen pflegt, was sie im übrigen auch sein und bedeuten mögen, nicht in Betracht kommen. Wenn die Kirche Gottes Offenbarung verkündigt, dann redet sie nicht auf Grund einer noch so tiefen und gläubigen Schau der Wirklichkeit der Welt und des Menschen, dann exegesiert sie nicht jene Ereignisse und Mächte, Gestalten und Wahrheiten, dann liest und erklärt sie, gebunden an ihren Auftrag und frei gemacht durch die damit empfangene Verheißung das Wort, das Jesus Christus heißt, und darum das Buch, das von ihm zeugt. Sie ist und sie bleibt dankbar für die Erkennbarkeit Gottes, in der er sich selber uns gegeben hat, indem er uns seinen Sohn gab» ${ }^{8}$.

Wie lässt sich das damalige Anliegen verstehen? Die Barmer Thesen stellen das den Menschen anredende Wort ins Zentrum, diesem sei zu vertrauen und ihm sei zu gehorchen. Ob dieser Aufruf zu Gehorsam eine totalitäre Forderung nach Unterordnung oder ein totales Bestimmen des Menschen zu seiner Freiheit ist, entscheidet sich am Verständnis des Wortes Gottes. Dieses Wort bedeutet im Rahmen der Barmer Thesen nicht Information oder Instruktion, sondern Anrede und Hinweis. Es spricht nicht so an, dass es überhört werden könnte und deshalb indoktriniert werden muss, sondern es scheint interessant genug zu sein, um selber auf sich aufmerksam zu machen. Wer es hört, lässt sich darauf ein und eignet es sich an, identifiziert sich damit. Wer es gehört hat, bleibt darauf ansprechbar, hört weiterhin auf es, gehorcht ihm gern. So ist es nicht eine Vorschrift, die befolgt würde, weil sie auch noch (neben anderem) hilfreich und vernünftig erscheint, sondern es wird im Gedächtnis behalten, weil es zum Leben ausreicht. Und während natürliche Theologie mit dem Wort Gottes einen Begründungsanspruch verbindet, kann und will die

K. Barth, Kurze Kommentierung des ersten Satzes, 87.Vgl. KD II/1, 200. 
Theologie der Barmer Thesen nicht erklären, weshalb etwas ist (und nicht vielmehr nichts), sondern will zu verstehen geben, wie, in welcher Weise etwas für einen Menschen so ist, wie es ist. Es geht nicht um eine große Überschau über Weltzusammenhänge, sondern bloß um das auf Gott ausgerichtete Da- und Sosein des Menschen in der Welt. Aber es geht dabei um alles, was den Menschen betrifft. Darin ist die Inanspruchnahme durch das Wort Gottes total. Einer auf Begründung abzielenden natürlichen Theologie (und ebenso einer Ideologie) geht es hingegen darum, den Menschen im Horizont bestimmter Annahmen als bloßen Gegenstand zu betrachten. Es geht ihr darum, alle Menschen unter dem von ihr bestimmten Gesichtspunkt zu sehen. Die Inanspruchnahme durch das so verstandene Wort Gottes ist dadurch totalitär. Sie sieht immer nur das, was sie sehen will und sieht damit immer nur das Gleiche, nämlich das, was ihr gleicht. Und was ihr nicht gleichen will, wird ihr gleichgemacht - oder vernichtet. Durch eine Theologie bzw. eine kirchlicheVerkündigung, die den Menschen angeht, weil sie ihn anspricht, wird hingegen der Mensch darauf hingewiesen, dass er das sieht, was ihm durch Jesus Christus gezeigt wird. Er sieht immer nur ihm durch Jesus Christus Zugewandtes, und was ihm nicht zugewandt ist, braucht er nicht herbeizuzwingen, sondern wird sich ihm früher oder später zuwenden.

\section{IV.}

Das Wort Jesus Christus bedeutet für Barth: Gott spricht den Menschen an. Wie ist das $\mathrm{zu}$ verstehen? Jesus Christus ist $\mathrm{zu}$ verstehen als die Norm adäquater Rede vom Menschen und zugleich als deren Inhalt, insofern nämlich Gott den Menschen als Menschen anspricht, indem er sich ihm zuwendet und ihn in seine Geschichte und Zukunft in Jesus Christus mit einbezieht. Durch dieses Angesprochenwordensein und -werden hat der Mensch teil an dem, was Gott tut. Wer sich der Geschichte und Zukunft Gottes anvertraut, gewinnt einen gewissen Abstand zu dem, was ihn ständig zum Handeln auffordert (zu sich selbst, seinen Mitmenschen und den Zusammenhängen, in denen er lebt). Sein Da- und Sosein ist, unabhängig davon, wie er auf diese Forderungen reagiert und ob er dem ihn Andrängenden gegenüber bestehen wird oder nicht, schon geborgen und aufgehoben; damit ist er schon versöhnt (mit Gott und damit mit sich und der Welt). Hat der Mensch Gott in Jesus Christus vertraut, wird er auch der Welt vertrauen können. Ob der Mensch hingegen noch einem 〈Gott〉 vertrauen wird, weil er der 
Welt vertraut bzw. zu vertrauen versucht hat, ist durchaus fraglich. Denn Vertrauen ist beständig durch die Meinung nicht nur beeinflusst, sondern bedroht, dass Kontrolle eben besser sei als Vertrauen. $\mathrm{Zu}$ vertrauen ist aber erst möglich, wenn der Mensch Kontrolle abgibt und damit Gott anstelle des eigenen Fürwahrhaltens vertraut. Der Mensch ist der Mensch, als den Gott ihn anspricht. Das befreit den Menschen davon, sich selber einen Namen machen zu müssen.

Vertrauen beinhaltet deshalb, theologisch verstanden, ein SichVerhalten in einem gewissen Abstand zu der Instanz, die einen jeweils zu handeln fordert. Dieses 〈Spiel〉 rührt daher, dass die in einem Gefühl usf. gründende oder im Zusammenhang von Herkunft, Rasse, Staatsordnung, Beruf usf. an einen gerichtete Forderung durch Gott bereits erfüllt und aufgehoben ist. Daran erinnert das Wort Gottes. Eine Theologie, die vom Menschen nicht so redet, wie Gott ihn angesprochen hat, sondern weiterhin Forderungen an ihn stellt, hat die Orientierung an Jesus Christus preisgegeben.

V.

Jesus Christus und keiner anderen Instanz ist im Glauben zu vertrauen, denn nur in diesem Blick eröffnen sich Vertrauensräume, die sich nicht plötzlich in Räuberhöhlen verwandeln. Im Blick auf einen Despoten kann keine Vertrauensbeziehung entstehen. Vertrauen ist unter despotischen, totalitären Bedingungen nicht möglich, weil es gar nicht aufrechterhalten werden kann. Vertrauen bedarf einer Ordnung, in der Menschen frei handeln können. Totalitäre Systeme beschneiden Freiheiten, indem sie sich über die Grenzen des Individuums hinwegsetzen. Sie üben dadurch ihre Herrschaft auch in Bereichen aus, die ihrem Wesen nach herrschaftslos funktionieren und keinem Zweck, sondern ausschließlich dem Menschen als Menschen dienen. Die I. These der Barmer Theologischen Erklärung hat daran erinnert, was die Aufgabe von Theologie und Kirche ist und wie in der Kirche (und durch ihre Glieder in der weiteren Öffentlichkeit) vom Menschen die Rede sein soll ${ }^{9}$, damit

\footnotetext{
9 Vgl. K. Barth,Theologische Existenz heute!, in:Theologische Existenz heute. Heft 1, München 1933, 40: «Sie [sc. die Kirche] ist die naturgemäße Grenze jedes, auch des totalen Staates. Denn das Volk lebt auch im totalen Staat vom Worte Gottes, dessen Inhalt ist: 〈Vergebung der Sünden, Auferstehung des Fleisches und ein ewiges Leben〉 Diesem Wort haben Kirche und Theologie zu dienen für das Volk.»Vgl. die V. Barmer These: Christen haben nicht dem Staat zu vertrauen und zu gehorchen, sondern Gott.
} 
dieser nicht in Angst und Schrecken ausharren muss, sondern in Vertrauen leben kann. ${ }^{10}$

- Marcel Egli arbeitet im Rahmen des Projekts «Vertrauen verstehen» an einer Dissertation mit dem Thema «Vertrauen und geschenkte Identität. Vertrauen in theologischer Perspektive im Anschluss an die Versöhnungslehre Karl Barths».

${ }^{10}$ Ich danke Simon Peng-Keller, der frühere Fassungen dieses Textes gelesen und kommentiert hat. 